\title{
ASPECTOS ECOLÓGICOS DE DOS CACTÁCEAS MEXICANAS AMENAZADAS: IMPLICACIONES PARA SU CONSERVACIÓN
}

\author{
Ricardo Álvarez, Héctor Godínez-Álvarez', Ulises Guzmán y Patricia Dávila \\ UBIPRO, FES-Iztacala, Universidad Nacional Autónoma de México. Av. de los Barrios 1, Los Reyes Iztacala, \\ Tlalnepantla, A.P. 314, Edo. de México 54090, México. \\ ${ }^{1}$ Autor para correspondencia. Tel 5623-1228, Fax 5623-1225, correo-e: chanogod@yahoo.com
}

\begin{abstract}
Resumen: Strombocactus disciformis y Turbinicarpus pseudomacrochele son dos especies de cactáceas mexicanas amenazadas. En este trabajo se evaluaron diversos aspectos ecológicos de estas especies como la estructura de tamaños, la ocupación del hábitat, la germinación de las semillas y la supervivencia de las plántulas. Además, se determinaron las actividades humanas que posiblemente podrían afectar el mantenimiento de las poblaciones. Los resultados mostraron que ambas especies presentan estructuras poblacionales en las que el número de individuos pequeños es bajo. Ocupan hábitats particulares y la germinación de las semillas, así como la supervivencia de las plántulas, son bajas. Además, están sujetas al efecto de diversas actividades humanas tales como la colecta ilegal de plantas y semillas, la ganadería y la extracción de materiales para construcción. De todas estas actividades, la colecta ilegal de semillas es aparentemente una de las principales amenazas para el mantenimiento de las poblaciones de estas especies.
\end{abstract}

Palabras clave: comercio ilegal, germinación, Strombocactus disciformis, supervivencia, Turbinicarpus pseudomacrochele.

\begin{abstract}
Strombocactus disciformis and Turbinicarpus pseudomacrochele are two threatened species of cacti endemic to Mexico. In this study different ecological aspects of these species such as size structure, habitat occupancy, seed germination, and seedling survival were analyzed. Additionally, human activities that could affect the population persistence were also determined. Results showed that both species have size structures with low numbers of small individuals. These species grow in particular habitats and the germination of their seeds as well as seedling survival were low. They are affected by various human activities such as illegal trade of seeds and plants, cattle grazing, and extraction of materials for construction. Of these, illegal trade apparently is one of the most important threats to population persistence of these species.
\end{abstract}

Key words: illegal trade, germination, Strombocactus disciformis, survivorship, Turbinicarpus pseudomacrochele.

$\mathbf{L}$ as cactáceas son un grupo de plantas con características biológicas y ecológicas particulares que las hacen vulnerables a diversos factores de perturbación naturales y humanos (Hernández y Godínez, 1994). Son plantas de lento crecimiento con ciclos de vida largos, que habitan sitios con condiciones edáficas específicas. El reclutamiento de nuevos individuos en las poblaciones es escaso y presentan patrones de distribución restringida (Hernández y Godínez, 1994; Godínez-Álvarez et al., 2003). Actualmente, una alta proporción de especies de cactáceas están amenazadas y han sido incluidas en listas internacionales relacionadas con la protección de la diversidad
(Hunt, 1999; Lüthy, 2001; SEMARNAP, 2001; IUCN, 2003).

Debido a esta situación, es necesario obtener información sobre diversos aspectos ecológicos de las especies amenazadas que proporcione un panorama general de su estado actual de conservación (Palmer, 1987; Schemske et al., 1994). Algunos de estos aspectos ecológicos son la distribución geográfica, la disponibilidad del hábitat, la estructura de tamaños y la reproducción de los individuos, entre otros. La estructura de tamaños proporciona información sobre la composición de las poblaciones en términos del sexo, la edad y la reproducción de los individuos; además, 
permite conocer si el reclutamiento en condiciones naturales ha ocurrido recientemente (Godínez-Álvarez et al., 2003). Por su parte, el análisis de la reproducción, particularmente de la germinación y la supervivencia de las plántulas, contribuye a conocer los factores que podrían afectar el reclutamiento de los individuos. De la misma manera, el estudio del hábitat ocupado por las especies y su disponibilidad en condiciones naturales permite determinar si la cantidad existente de sitios adecuados para la supervivencia y el crecimiento es un factor limitante del reclutamiento (Palmer, 1987).

Junto con la información sobre los aspectos ecológicos, también es necesario reconocer las actividades humanas que podrían afectar el mantenimiento de las poblaciones de las distintas especies de cactáceas amenazadas (Arias, 1993; Anderson et al., 1994; Lüthy, 2001). Algunas de estas actividades como la colecta excesiva de plantas y semillas, la agricultura, la ganadería y la erosión del suelo, podrían disminuir la fecundidad y/o la supervivencia de los individuos, afectando el reclutamiento. El análisis de los efectos de estas actividades contribuye a tener un panorama más completo y detallado del estado de conservación de las especies.

Toda esta información representa una base importante para la realización de estudios ecológicos más detallados, o bien para la toma de decisiones relacionadas con estas especies. Dicha información puede ser empleada para llevar a cabo estudios demográficos o monitoreos para cuantificar los cambios ocurridos en las poblaciones a lo largo del tiempo. Además, esta información puede proporcionar evidencia que permita apoyar la inclusión o exclusión de las especies amenazadas en las listas internacionales.

En este trabajo se evaluaron algunos aspectos ecológicos de Strombocactus disciformis (DC.) Britton et Rose y Turbinicarpus pseudomacrochele (Backeb.) Buxb. et Backeb., dos especies de cactáceas endémicas de México. Actualmente, estas especies están incluidas en el Apéndice I de la CITES (Hunt, 1999) y están consideradas como amenazadas y en peligro de extinción, respectivamente, en la Norma Oficial Mexicana (SEMARNAP, 2001). Estas especies presentan un patrón restringido de distribución geográfica y se encuentran amenazadas principalmente por el cambio en el uso de suelo y la colecta ilegal (Anderson et al., 1994; Lüthy, 2001). Los aspectos ecológicos evaluados fueron la estructura de tamaños, el hábitat ocupado por las especies, la germinación de las semillas y la supervivencia de las plántulas. Además, con base en observaciones de campo e información obtenida en internet y dependencias gubernamentales, se determinaron las actividades humanas que afectan las poblaciones de estas especies. Con esta información se pretende contribuir al conocimiento del estado actual de conservación de ambas especies y los posibles factores que afectan su mantenimiento.

\section{Materiales y métodos}

Para la realización de este trabajo se seleccionaron tres poblaciones distintas para cada una de las especies. En el caso de $S$. disciformis, las poblaciones fueron Vizarrón, Peña Blanca 1 y Peña Blanca 2, en el estado de Querétaro. Para T. pseudomacrochele, las poblaciones fueron Bernal en Querétaro, así como Ixmiquilpan y Zimapán en Hidalgo. En esta última especie, los individuos de las poblaciones de Bernal e Ixmiquilpan pertenecen a la subespecie pseudomacrochele, en tanto que los de Zimapán a la subespecie krainzianus. En términos generales, todos estos sitios están ubicados en altitudes que varían de 1,432 a 2,091 m snm. El clima es cálido con lluvias en verano. La temperatura y la precipitación total promedio anuales son $350-750 \mathrm{~mm}$ y $16-22^{\circ} \mathrm{C}$. Los suelos son de origen calcáreo y el principal tipo de vegetación es el matorral xerófilo en el que Hechtia glomerata Zucc., Jatropha dioica Sessé ex Cerv., Larrea tridentata (Sessé et Moc. ex DC.) Coville, Myrtillocactus geometrizans (Mart.) Console, Opuntia imbricata (Haw.) DC. y Prosopis glandulosa Torr. son algunas de las especies dominantes.

S. disciformis y T. pseudomacrochele son cactáceas globosas con tallos pequeños de 5-10 y 2-3 cm de diámetro, respectivamente (Anderson et al., 1994; Lüthy, 2001). Las flores de $S$. disciformis son blanco cremosas y son producidas a principios de la primavera. Son diurnas y permanecen abiertas por varios días, siendo polinizadas principalmente por abejas. Los frutos son cilíndricos, cafés y dehiscentes. Las semillas son pequeñas, rojizas, tuberculadas y con estrofiolo. Debido a lo anterior, se ha sugerido que las hormigas son los principales dispersores de las semillas (Bregman, 1988). Los individuos de esta especie crecen comúnmente en laderas inclinadas de origen calcáreo. Por otra parte, las flores de T. pseudomacrochele pueden ser de color rosa pálido o amarillo con una raya central de color rosa oscuro. Son diurnas y polinizadas por abejas. Los frutos maduros son esféricos, rojizos y dehiscentes. Las semillas son pequeñas, negras y tuberculadas. Esta especie crece en laderas con pendientes poco inclinadas, en suelos y rocas de origen calcáreo.

Estructura de tamaños. Durante enero y febrero de 2002 se establecieron 10-20 parcelas de $1 \mathrm{~m}^{2}$ por especie para cada una de las poblaciones estudiadas. Estas parcelas se ubicaron en los sitios en donde se encontró creciendo el mayor número de individuos, tratando de abarcar la mayor proporción del área. En cada parcela se ubicaron todos los individuos y se midió el diámetro de cada uno de sus tallos utilizando un vernier digital. Con estos datos se calculó el diámetro total de cada individuo como la suma de los diámetros de todos sus tallos. Los individuos fueron clasificados con base en el diámetro total en distintas categorías de tamaño, las cuales fueron definidas considerando el 
intervalo de los datos y el número total de individuos. Para S. disciformis, las categorías empleadas fueron (cifras en $\mathrm{mm})$ : 0-5, 5-10, 10-15, 15-20, 20-25, 25-30, 30-35, 35-40, 40-45, 45-50 y > 50. En el caso de T. pseudomacrochele las categorías utilizadas fueron (en mm) : 0-10, 10-20, 20-30, $30-40,40-50,50-60,60-70,70-80,80-90,90-100,100-$ $150,150-200,200-250$ y 250-300. Las estructuras de tamaños fueron comparadas con una prueba de $\chi^{2}$ para determinar si existían diferencias significativas entre las poblaciones de cada especie. Sin embargo, para T. pseudomacrochele no fue posible realizar dicha prueba estadística debido al bajo número de plantas encontrado en algunas poblaciones.

Ocupación del hábitat. El hábitat ocupado por ambas especies se determinó con base en censos realizados en campo para estimar la disponibilidad de distintos tipos de hábitat. Para esto, en cada localidad se utilizaron entre 2 y 4 líneas de Canfield de 10-30 m, en las que se registró la longitud ocupada por: a) macizos rocosos con grietas, b) suelo desprovisto de vegetación, c) suelo cubierto por hierbas y d) suelo cubierto por arbustos. Dichas líneas se ubicaron en los mismos sitios en donde crecen las plantas y en los que se realizaron las parcelas de $1 \mathrm{~m}^{2}$ para determinar la estructura de tamaños. Con base en la longitud ocupada por cada hábitat con respecto a la longitud total de la línea de Canfield, se calculó la proporción de los distintos hábitats disponibles para las plantas. Para conocer si los individuos ocupaban los hábitats de manera proporcional a su disponibilidad, se contó el número de individuos existente en cada uno de los hábitats empleando las parcelas de $1 \mathrm{~m}^{2}$ mencionadas anteriormente. Con la proporción disponible de los distintos hábitats y el número total de plantas contadas, se estimó el número de individuos que se esperaría encontrar si la distribución fuera al azar. Estos valores se compararon con el número de plantas observado mediante una prueba de $\chi^{2}$. Para evaluar las diferencias entre los distintos hábitats se calcularon los residuos ajustados (Everitt, 1977).

Germinación y supervivencia. Para evaluar la germinación de las semillas y la supervivencia de las plántulas se realizaron experimentos en laboratorio y campo, respectivamente. Se colectaron 10-22 frutos maduros de distintos individuos en las diferentes poblaciones de cada una de las especies. Las semillas se obtuvieron por disección y fueron desinfectadas con una solución de hipoclorito de sodio (30\%) durante $5 \mathrm{~min}$; posteriormente fueron lavadas con agua destilada y secadas a temperatura ambiente.

En el experimento de germinación, la unidad experimental consistió de una caja Petri con papel filtro y 25 semillas. Para cada población se realizaron ocho repeticiones que se colocaron en una cámara ambiental con temperatura constante de $25^{\circ} \mathrm{C}$, luz fluorescente y fotoperiodo de $12 \mathrm{~h}$. Las cajas se revisaron diariamente para registrar el número de semillas germinadas. Una semilla se consideró germinada cuando emergía la radícula. La proporción de semillas germinadas se comparó entre las distintas poblaciones de cada especie con un análisis de la varianza de una vía, previa transformación arcoseno de los datos. Además de la proporción de semillas germinadas, para cada población se calculó el índice de germinación de Scott mediante la fórmula: IG = $\Sigma\left(\mathrm{n}_{i} \mathrm{t}_{i}\right) / \mathrm{N}$, en donde $\mathrm{n}_{i}$ es el número de semillas germinadas el día $i, \mathrm{t}_{i}$ es el número de días transcurridos desde el inicio del experimento y $\mathrm{N}$ es el número total de semillas germinadas. De acuerdo con este índice, entre mayor es el valor calculado, mayor es la velocidad a la que ocurre la germinación de las semillas (González-Zertuche y OrozcoSegovia, 1996). Para cada especie, los índices de germinación fueron comparados con una prueba de KruskalWallis para determinar si existían diferencias significativas entre las poblaciones.

Las plántulas obtenidas en el experimento de germinación fueron empleadas para evaluar la supervivencia en campo. En el caso de T. pseudomacrochele, las plántulas fueron transplantadas dos meses después de la germinación a charolas de plástico con suelo colectado en cada una de las poblaciones. El procedimiento en $S$. disciformis fue distinto debido a que el tamaño pequeño de las plántulas dificultaba su transplante. Por esta razón, las semillas fueron sembradas directamente en charolas de plástico con suelo de cada población para obtener las plántulas. Las charolas se colocaron en una cámara ambiental con las mismas condiciones empleadas en el experimento de germinación. La unidad experimental para evaluar la supervivencia consistió de una charola con 30 plántulas para T. pseudomacrochele y 50 plántulas para $S$. disciformis. Para cada población se realizaron dos repeticiones, las cuales fueron transportadas a uno de los sitios de estudio de cada especie durante los primeros días de septiembre de 2002. En S. disciformis, todas las repeticiones fueron transportadas a Peña Blanca 1 , en tanto que en $T$. pseudomacrochele se transportaron a Zimapán. Esta decisión permitió disminuir el riesgo de pérdida de las repeticiones y evitar problemas logísticos. Las plántulas se aclimataron antes de ser llevadas al campo incrementando su exposición a la radiación solar y disminuyendo la frecuencia de riego durante dos meses. En el campo, las charolas se colocaron debajo de arbustos de Larrea tridentata de tamaño similar, tratando de simular la protección brindada por las grietas de las rocas durante estas etapas del ciclo de vida. Esta decisión se tomó debido a la imposibilidad de transplantar las plántulas de ambas especies a las grietas existentes en las rocas. Cada 15-30 días se realizaron censos para contar el número de supervivientes y los datos se analizaron con la prueba de Peto y Peto (Pike y Thompson, 1986).

Impacto de la actividad humana. El impacto de la actividad humana sobre ambas especies se determinó con base en 
información proporcionada por la Procuraduría Federal de Protección al Ambiente (PROFEPA), así como observaciones en campo para evaluar el posible efecto de actividades como la agricultura, la ganadería caprina y la extracción de materiales, entre otros. Además, esta información se complementó con una búsqueda en internet para contar el número de portales directamente relacionados con estas dos especies. La búsqueda se realizó en Yahoo! empleando los nombres científicos de las especies. Los portales encontrados fueron revisados y clasificados de acuerdo con su contenido en cinco categorías: (1) semillas, portales que venden semillas o que proporcionan información relacionada con las condiciones adecuadas para su germinación; (2) localidades, portales que proporcionan información sobre las localidades en las que crecen las especies; (3) fotos, portales con material fotográfico de plantas silvestres y cultivadas, así como descripciones morfológicas de las especies; (4) plantas, portales en donde se venden plantas; y (5) otros, portales que proporcionan información sobre diversos aspectos como técnicas de cultivo, alcaloides presentes en los tejidos, tipos de abono empleados para estimular el crecimiento, etc.

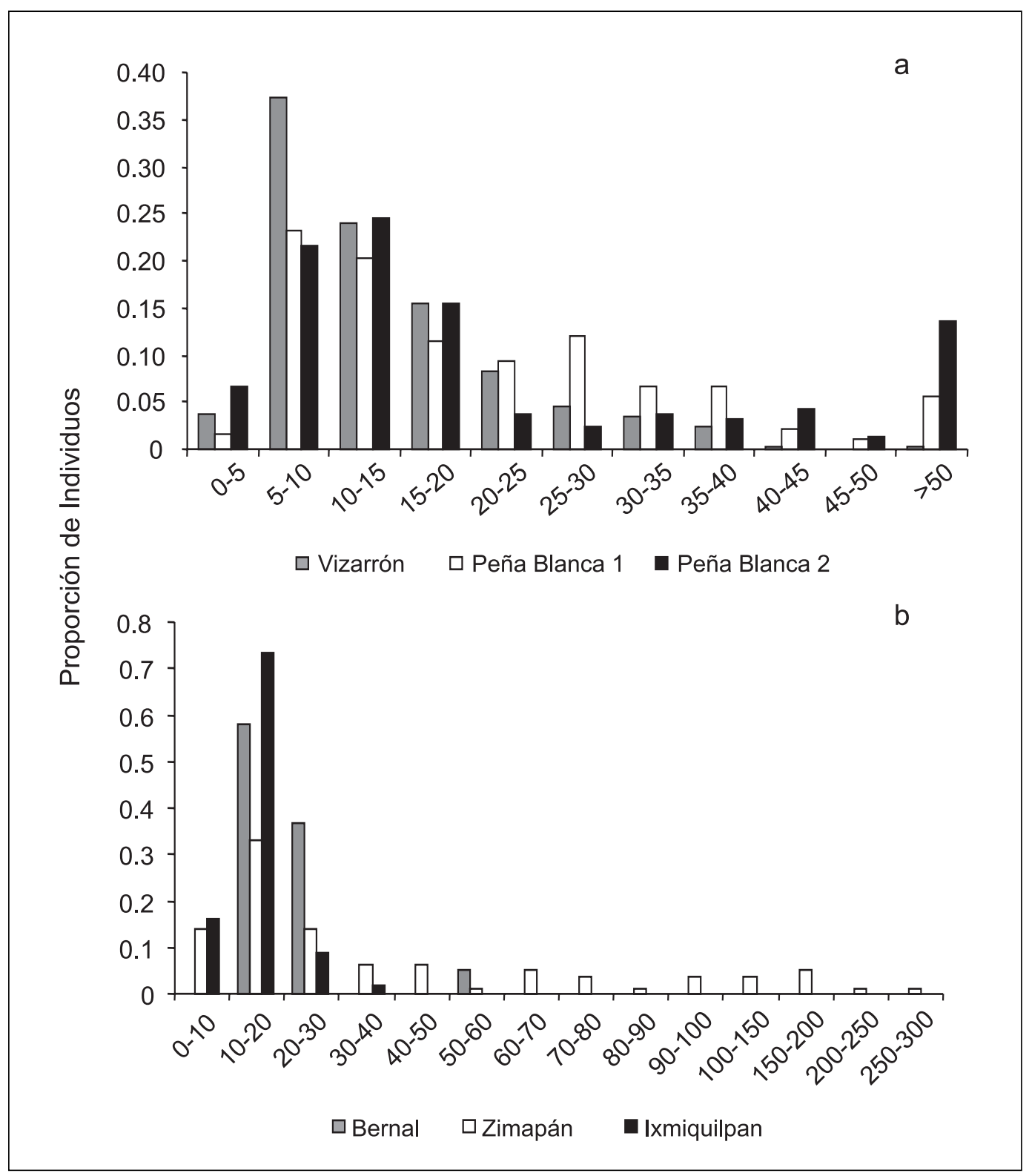

Figura 1. Estructuras de tamaños de Strombocactus disciformis (a) y Turbinicarpus pseudomacrochele (b) en tres poblaciones diferentes. 


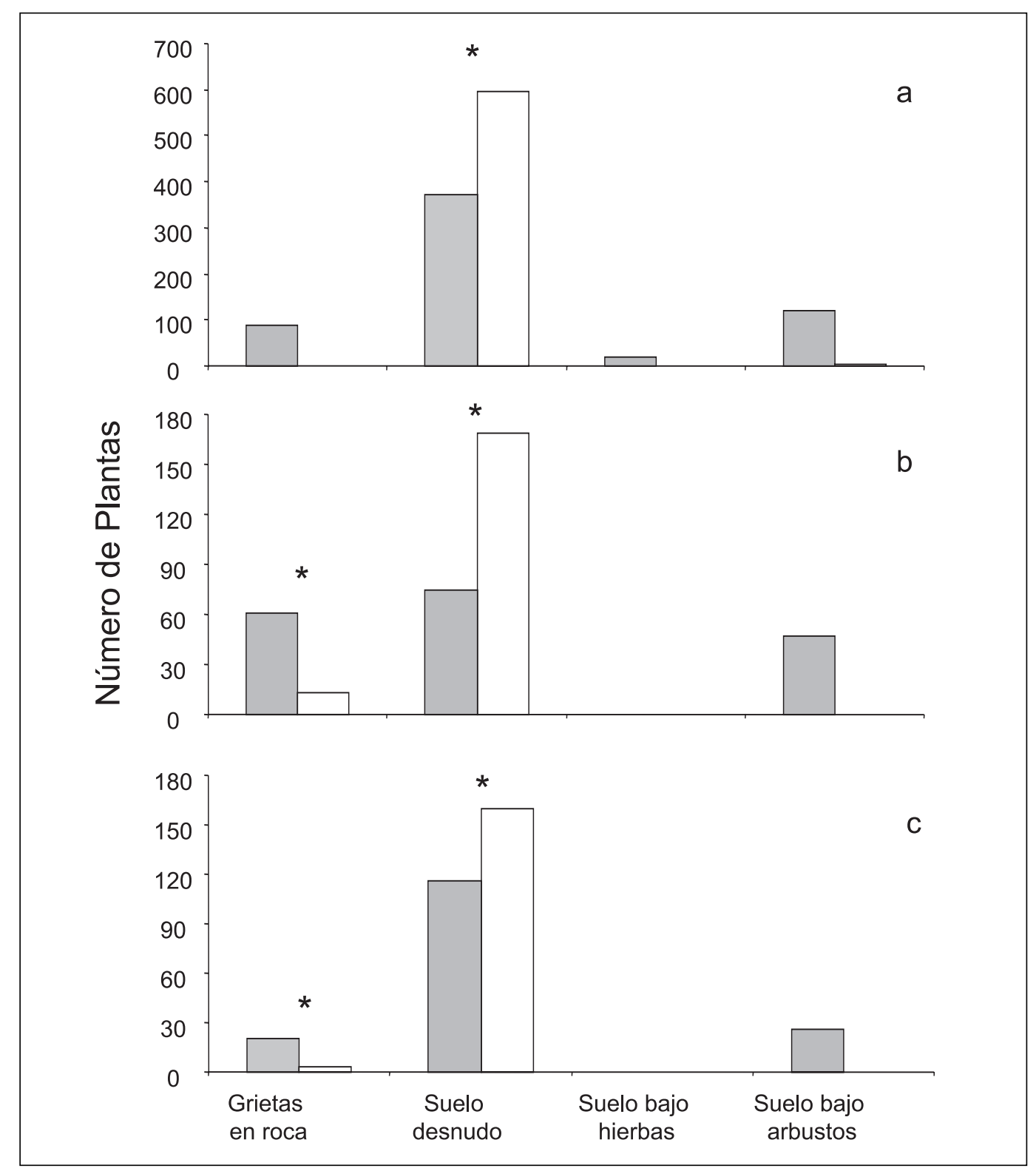

Figura 2. Hábitats ocupados por S. disciformis en tres poblaciones diferentes: a) Vizarrón, b) Peña Blanca 1 y c) Peña Blanca 2. Las barras de color blanco y gris se refieren al número de plantas observado y esperado por azar. El asterisco indica diferencias significativas $(P<0.05)$.

\section{Resultados}

Estructura de tamaños. Los resultados obtenidos muestran que las poblaciones de $S$. disciformis difieren significativamente en su estructura de tamaños $\left(\chi^{2}=148.18\right.$, g.1. $=20$, $P<0.0001)$. Todas las poblaciones tienen una baja proporción de individuos $<5 \mathrm{~mm}$ de diámetro $(2-7 \%)$. De la misma manera, la mayoría de los individuos que las conforman están comprendidos en las categorías de 5-20 mm.
Sin embargo, existen diferencias en la proporción de individuos > $40 \mathrm{~mm}$. Las poblaciones de Peña Blanca 1 y 2 tienen una proporción de 9-20\%, en tanto que la población de Vizarrón únicamente presentó $0.3 \%$ (figura 1a).

La estructura de tamaños de T. pseudomacrochele también fue diferente entre las poblaciones estudiadas (figura 1b). En las poblaciones de Bernal e Ixmiquilpan, las categorías de 0-30 $\mathrm{mm}$ de diámetro fueron las únicas que presentaron individuos (60-90\%). Por el contrario, la 


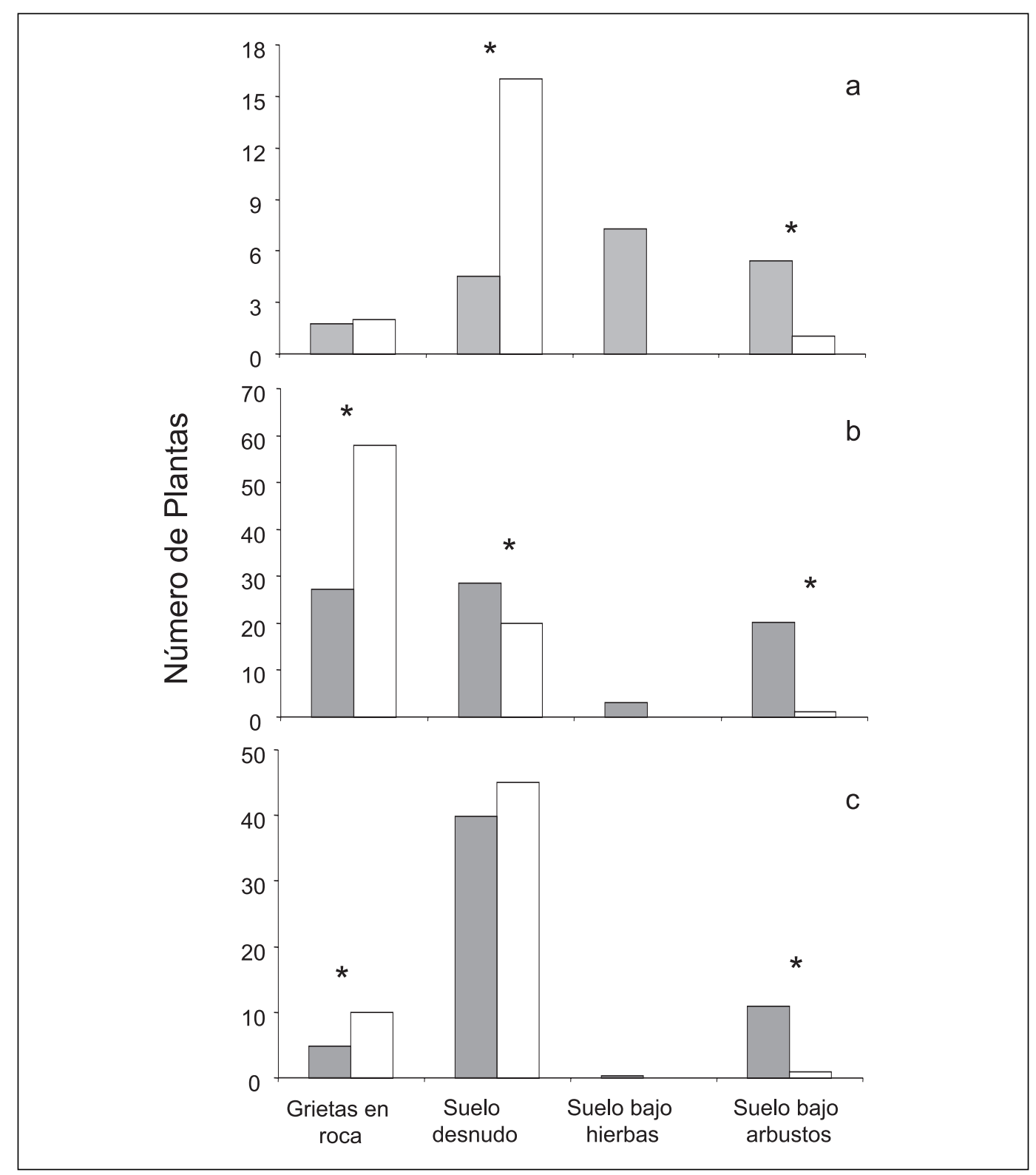

Figura 3. Hábitats ocupados por T. pseudomacrochele en tres poblaciones diferentes: a) Bernal, b) Zimapán y c) Ixmiquilpan. Las barras de color blanco y gris se refieren al número de plantas observado y esperado por azar. El asterisco indica diferencias significativas $(P<0.05)$.

población de Zimapán presentó individuos en todas las categorías de tamaño, aunque la mayor proporción $(60 \%)$ también se observó en las categorías de 0-30 mm. En todas las poblaciones, el número de individuos $<10 \mathrm{~mm}$ fue bajo. No obstante las diferencias anteriores, es importante recordar que éstas no pudieron ser evaluadas estadísticamente debido al bajo número de plántulas encontrado.

Ocupación del hábitat. La proporción de los distintos hábitats considerados fue relativamente similar en las tres pobla- ciones de $S$. disciformis. El suelo desprovisto de vegetación presentó la mayor proporción (41-71\%), seguido de las grietas en macizos rocosos (13-33\%), el suelo cubierto por arbustos $(16-26 \%)$ y hierbas $(0-3 \%)$. En todas las poblaciones hubo diferencias significativas en la distribución observada y esperada de los individuos, de acuerdo con la proporción ocupada por los distintos hábitats (Vizarrón: $\chi^{2}$ $=3678.62$, g.1. $=3, P<0.001$; Peña Blanca 1: $\chi^{2}=488.51$, g.l. $=2, P<0.001$; Peña Blanca $2: \chi^{2}=122.12$, g.l. $=2, P$ $<0.001)$. Se encontró que los individuos crecen más fre- 


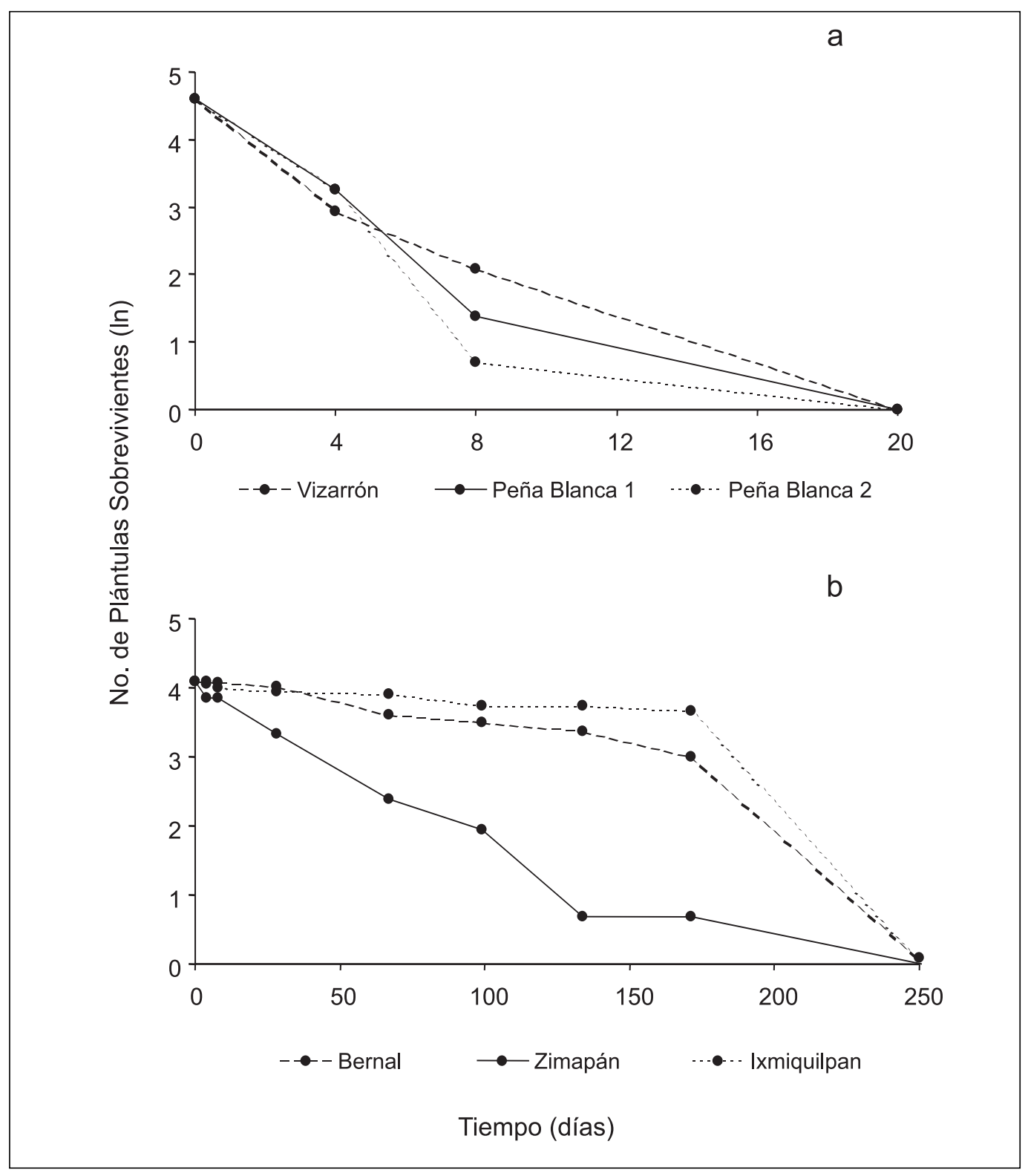

Figura 4. Curvas de supervivencia de las plántulas de Strombocactus disciformis (a) y Turbinicarpus pseudomacrochele (b), emergidas de semillas colectadas en tres poblaciones diferentes. El experimento inició en los primeros días de septiembre de 2002, llevándose a cabo en Peña Blanca 1 para S. disciformis y en Zimapán para T. pseudomacrochele.

cuentemente en suelo desprovisto de vegetación en comparación con el resto de los hábitats (figura 2).

En T. pseudomacrochele, los hábitats disponibles variaron según las distintas poblaciones. En Bernal, el suelo cubierto por hierbas ocupó la mayor proporción (38\%), seguido por el suelo cubierto por arbustos (29\%) y el suelo desprovisto de vegetación (24\%). Por otra parte, en Ixmiquilpan y Zimapán el suelo desprovisto de vegetación (36-71\%), el suelo cubierto por arbustos (19-26\%) y las grietas en rocas
(9-34\%) fueron los hábitats más comunes. El número de individuos observado en los diferentes hábitats fue distinto del esperado por azar (Bernal: $\chi^{2}=38.8$, g.l. $=3, P<$ 0.001; Zimapán: $\chi^{2}=263.75$, g.l. $=3, P<0.001$; Ixmiquilpan: $\chi^{2}=32.7$, g.1. $=3, P<0.001$ ). En Bernal, la mayoría de los individuos crecen en suelo desprovisto de vegetación, mientras que en Zimapán e Ixmiquilpan se encuentran en grietas de rocas (figura 3 ). 
Germinación y supervivencia. Las poblaciones de $S$. disciformis variaron en el índice de germinación $\left(\chi^{2}=20.2\right.$, g.l. $=2, P<0.001)$ y la proporción de semillas germinadas $(\mathrm{F}=$ 66.5, g.l. $=2,21, P<0.0001)$. Las semillas colectadas en Peña Blanca 2 presentaron los mayores valores (promedios \pm E.E.: $4.9 \pm 0.24,0.82 \pm 0.05)$, seguidas por las de Peña Blanca $1(3.27 \pm 0.23,0.56 \pm 0.09)$ y Vizarrón $(1.45 \pm 0.19$, $0.25 \pm 0.07)$. No obstante estas diferencias, la supervivencia de las plántulas en campo fue similar, independientemente de la población de origen ( $\mathrm{LR}=1.1$, d.f. $=2, P>0.05)$. Las plántulas sobrevivieron un tiempo muy corto debido a que después de 20 días no existía ninguna (figura 4a).

De igual forma, las poblaciones de T. pseudomacrochele también fueron diferentes en el índice de germinación $\left(\chi^{2}\right.$ $=17.2$, d.f. $=2, P<0.001)$ y en la proporción de semillas germinadas $(\mathrm{F}=19.9$, d.f. $=2,21, P<0.001)$. Las semillas con los mayores $(5.92 \pm 0.37,0.67 \pm 0.08)$ y menores $(2.52$ $\pm 0.2,0.38 \pm 0.08)$ valores fueron las de Zimapán e Ixmiquilpan, respectivamente. Por su parte, la población de Bernal presentó valores intermedios $(3.08 \pm 0.23,0.49 \pm$ 0.09). Con respecto a la supervivencia, las plántulas de Bernal e Ixmiquilpan permanecieron vivas por más de 150 días, en tanto que las de Zimapán presentaron una mortalidad relativamente constante desde el inicio del experimento (figura $4 b$ ). Estas diferencias fueron significativas ( $\mathrm{LR}=$ 115.2, d.f. $=2, P<0.001)$.

Impacto de la actividad humana. La información proporcionada por la PROFEPA mostró que ambas especies son colectadas ilegalmente en nuestro país, debido a que varios cargamentos han sido asegurados en diferentes estados de la República Mexicana y otros países. Por ejemplo, entre 1997 y 2001 fueron detenidos tres cargamentos que contenían 1-70 plantas de $S$. disciformis en México (Morelos y Nuevo León) y Holanda. De manera similar, en el periodo comprendido entre 1996 y 2001, cuatro cargamentos con 4279 individuos de T. pseudomacrochele fueron asegurados en los estados de Morelos y Nuevo León o repatriados de otros países como Holanda y la República Checa.

Además de la colecta ilegal de individuos, las observaciones en campo mostraron que existen otras actividades humanas que podrían afectar las poblaciones de ambas especies. En el caso de $S$. disciformis, se observaron hatos de chivos en las poblaciones analizadas, por lo que el pastoreo y el pisoteo del ganado podrían afectar la supervivencia. Con respecto a $T$. pseudomacrochele, se encontraron evidencias de la colecta ilegal de semillas en las poblaciones de Bernal e Ixmiquilpan. En estas poblaciones se observó que varias plantas carecían de ápice, el cual fue removido junto con los frutos inmaduros por un grupo de alemanes encontrado en ambas localidades. Asimismo, otras actividades como la extracción de materiales y el pastoreo del ganado caprino también pueden afectar a los individuos de esta especie, particularmente los de Bernal.
En internet se encontraron en total 161 portales con información de $S$. disciformis y 106 de $T$. pseudomacrochele. En ambas especies, los portales encontrados pertenecen a países como Alemania, Estados Unidos, Francia, Inglaterra, Italia y Japón, entre otros. Para S. disciformis, solamente 77 portales pudieron ser clasificados en las distintas categorías debido a que el resto no aportaba información relevante o estaba en idiomas como ruso, japonés o checo. La mayoría de estos portales contenía fotos pertenecientes a colecciones particulares $(52 \%)$, así como información para la venta de plantas $(17 \%)$ y semillas $(17 \%)$. Asimismo, se encontraron dos portales con información detallada de las localidades en las que crece esta especie. Con respecto a $T$. pseudomacrochele, únicamente 61 portales del total encontrado pudieron ser clasificados. La mayoría de éstos contenía fotos $(44 \%)$ e información para la venta de plantas $(28 \%)$ y semillas (16\%). Solamente se encontró un portal con información de las localidades en donde crece esta especie.

\section{Discusión}

En términos generales, los resultados obtenidos muestran que $S$. disciformis y $T$. pseudomacrochele presentan características ecológicas que las hacen vulnerables a distintos factores de disturbio. Ambas especies presentan estructuras poblacionales en las que el número de individuos pequeños es bajo y ocupan hábitats particulares. Tanto la germinación de las semillas como la supervivencia de las plántulas son bajas. Además estas especies, populares entre los coleccionistas, están sujetas al efecto de diversas actividades humanas.

El análisis de las estructuras de tamaños de ambas especies mostró que la proporción de individuos pequeños es baja en todas las poblaciones analizadas. Este resultado sugiere que el reclutamiento es un proceso poco frecuente que podría depender de factores físicos como la temperatura y la precipitación y/o de factores biológicos como la reproducción y las interacciones con otros organismos (Brum, 1973; Parker, 1989). Otras especies de cactáceas con distintas formas de crecimiento también se caracterizan por presentar estructuras de tamaños de este tipo (GodínezÁlvarez et al., 2003). El reclutamiento de nuevos individuos en las cactáceas es afectado fuertemente por las condiciones microambientales. En este sentido, se ha sugerido que los sitios adecuados para la germinación y la supervivencia están ubicados debajo de la copa de plantas perennes o en grietas de rocas (Sosa y Fleming, 2002). En estos sitios la radiación solar disminuye, por lo que la humedad disponible en el suelo aumenta, favoreciendo la germinación de las semillas (Rojas-Aréchiga y Vázquez-Yanes, 2000; Sosa y Fleming, 2002). Además, el riesgo de depredación por vertebrados e invertebrados también puede disminuir, incrementando la probabilidad de supervivencia de las semillas y las plántulas (Sosa y Fleming, 2002). 
S. disciformis y T. pseudomacrochele crecen preferentemente en suelo desprovisto de vegetación y en grietas presentes en las rocas. En el caso de $S$. disciformis, los sitios ocupados por los individuos se encontraron únicamente en laderas de origen calcáreo con pendientes pronunciadas, por lo que las condiciones necesarias para la supervivencia y el crecimiento son muy específicas. Otras cactáceas globosas como Echinocereus pulchellus, Mammillaria gaumeri, M. magnimamma y Turbinicarpus pseudopectinatus (Martínez et al., 1994; Leirana-Alcocer y ParraTabla, 1999; Valverde et al., 1999, Navarro y FloresMartínez, 2002), también han sido encontradas creciendo en grietas de rocas. La frecuente ocupación de estos hábitats por ambas especies puede deberse a una dispersión limitada de las semillas y al hecho de que en estos sitios probablemente se encuentran las condiciones adecuadas para el reclutamiento. Bregman (1988), basado en las características morfológicas de las semillas de $S$. disciformis, sugirió que las hormigas eran los principales dispersores de las semillas de esta especie. Sin embargo, en condiciones naturales no se observó ningún insecto que potencialmente pudiera realizar dicha actividad. Los frutos de $S$. disciformis y T. pseudomacrochele son dehiscentes, por lo que es posible suponer que algún factor abiótico como el agua, el viento o la gravedad podría ser el agente dispersor.

La germinación de las semillas y la supervivencia de las plántulas de las dos especies fue baja en condiciones de laboratorio y campo, respectivamente. La germinación occurrió en los primeros tres o cuatro días, por lo que es posible suponer que este proceso depende únicamente de la existencia de condiciones adecuadas de humedad (RojasAréchiga y Vázquez-Yanes, 2000). Sin embargo, el tiempo requerido para alcanzar $50 \%$ de germinación fue de hasta 12 y 18 días en $S$. disciformis y $T$. pseudomacrochele, respectivamente. Asimismo, la proporción de semillas germinadas varió de $25-82 \%$ en S. disciformis y de $38-67 \%$ en T. pseudomacrochele. Estos resultados son relativamente inferiores a los reportados para otras especies de cactáceas (Godínez-Álvarez y Valiente-Banuet, 1998; RojasAréchiga y Vázquez-Yanes, 2000). En condiciones naturales, una baja tasa y una baja proporción de semillas germinadas incrementan el riesgo de depredación de las semillas, disminuyendo la probabilidad de reclutamiento de los individuos. La alta variación observada en la proporción final de semillas germinadas en ambas especies puede estar relacionada con diferencias ambientales y genéticas existentes entre las poblaciones (Navarro y Guitián, 2003). En T. pseudomacrochele, las diferencias en el comportamiento germinativo también podrían estar relacionadas con la presencia de las distintas subespecies. Las semillas de la subespecie krainzianus (i.e. Zimapán) tuvieron mayores índices y porcentajes de germinación que las de la subespecie pseudomacrochele (i.e. Bernal e Ixmiquilpan).

Con respecto a la supervivencia, las plántulas de ambas especies permanecieron vivas por un corto periodo en condiciones de campo (i.e. 20 días en T. pseudomacrochele y 250 días en $S$. disciformis). Este mismo patrón de supervivencia ha sido descrito para otras especies de cactáceas con distintas formas de crecimiento (Godínez-Álvarez et al., 2003). La capacidad para almacenar agua es un factor fundamental para la supervivencia de las cactáceas en los ecosistemas áridos. Esta capacidad de almacenaje es reducida en las plántulas debido a que su superficie es mayor que su volumen, favoreciéndose la pérdida de agua por evapotranspiración (Nobel, 1994). La baja supervivencia de $S$. disciformis puede estar relacionada con este hecho, ya que las plántulas recién emergidas miden tan sólo ca. $0.5 \mathrm{~mm}$ de alto. La supervivencia en $T$. pseudomacrochele también podría ser afectada por el tamaño pequeño de las plántulas. Sin embargo, es importante resaltar que las distintas subespecies presentaron diferencias significativas en este atributo demográfico. Las plántulas de la subespecie krainzianus tuvieron una menor supervivencia que las de la subespecie pseudomacrochele. Aparentemente, estas diferencias no están relaciondas con el tamaño de las plántulas de ambas subespecies, ya que éstas son relativamente similares. Sin embargo, es necesario obtener datos para confirmar estas ideas.

El análisis de las actividades humanas mostró que la transformación del hábitat y el comercio ilegal son algunos de los principales factores que podrían afectar la permanencia de las poblaciones de $S$. disciformis y $T$. pseudomacrochele. El comercio ilegal puede ser considerado como una de las principales amenazas debido a que en internet es posible encontrar una gran cantidad de información sobre estas especies. Inclusive, es posible conocer las localidades exactas en donde crecen estas plantas en nuestro país. Debido a lo anterior, actualmente las poblaciones de ambas cactáceas son frecuentemente visitadas por extranjeros para colectar frutos y semillas (R. Álvarez y $\mathrm{H}$. Godínez-Álvarez, obs. pers.). La biología de estas especies es ampliamente conocida, ya que las poblaciones son visitadas precisamente durante los meses en los que se presenta la floración y la fructificación. La colecta ilegal de semillas tiene efectos negativos sobre el reclutamiento pues disminuye el potencial reproductivo de los individuos. Esta situación es particularmente crítica en $T$. pseudomacrochele, debido a que el número de semillas por fruto es ca. 30 (datos no mostrados en este trabajo).

En resumen, los resultados obtenidos sugieren que $S$. disciformis y $T$. pseudomacrochele presentan un bajo reclutamiento de nuevos individuos en sus poblaciones. El reclutamiento se presenta en hábitats particulares, cuya disponibilidad aparentemente no está limitada en condiciones naturales. Sin embargo, la germinación de las semillas y la supervivencia de las plántulas son bajas. Estas etapas del ciclo de vida son afectadas principalmente por las condiciones ambientales existentes en las poblaciones, aunque 
posiblemente también por diferencias genéticas. Las características ecológicas mencionadas determinan que estas especies sean vulnerables a distintas actividades humanas. La colecta ilegal de las semillas, la ganadería y la extracción de materiales para construcción son algunas de las principales amenazas para el mantenimiento de las poblaciones de estas especies. Actualmente, $S$. disciformis y $T$. pseudomacrochele están consideradas como amenazadas y en peligro de extinción, respectivamente, en la Norma Oficial Mexicana. Con base en la información obtenida en este trabajo, estas especies deberían permanecer en estas categorías para así tratar de protegerlas del efecto negativo de las actividades humanas. No obstante, esta decisión debería de ir acompañada de acciones encaminadas a disminuir dichas actividades, particularmente la colecta ilegal de las semillas. Finalmente, los resultados obtenidos también sugieren que en el caso de algunas especies de cactáceas como T. pseudomacrochele, los niveles taxonómicos infraespecíficos deben ser considerados en la instrumentación de las actividades de conservación.

\section{Agradecimientos}

Guillermo Sánchez de la Vega y Carlos Morin Valdés proporcionaron ayuda en el trabajo de campo. Este estudio fue financiado por la Comisión Nacional para el Conocimiento y Uso de la Biodiversidad (CONABIO), a través del proyecto V039.

\section{Literatura citada}

Anderson E.F., Arias S. y Taylor N.P. 1994. Threatened Cacti of Mexico. Vol. II. Royal Botanic Gardens, Kew.

Arias M.S. 1993. Cactáceas: Conservación y diversidad en México. Revista de la Sociedad Mexicana de Historia Natural XLIV:109-115.

Bregman R. 1988. Forms of seed dispersal in Cactaceae. Acta Botanica Neerlandica 37:395-402.

Brum G.D. 1973. Ecology of the saguaro (Carnegiea gigantea): phenology and establishment in marginal populations. Madroño 22:194-204.

Everitt B.S. 1977. The Analysis of Contingency Tables. Chapman and Hall, Londres.

Godínez-Alvarez H. y Valiente-Banuet A. 1998. Germination and early seedling growth of Tehuacan Valley cacti species: the role of soils and seed ingestion by dispersers on seedling growth. Journal of Arid Environments 39:21-31.

Godínez-Alvarez H., Valverde T. y Ortega-Baes P. 2003. Demographic trends in the Cactaceae. Botanical Review 69:173-203.

González-Zertuche L. y Orozco-Segovia A. 1996. Métodos de análisis de datos en la germinación de semillas, un ejemplo: Manfreda brachystachya. Boletín de la Sociedad Botánica de México 58:15-30.
Hernández M.H. y Godínez A.H. 1994. Contribución al conocimiento de las cactáceas mexicanas amenazadas. Acta Botanica Mexicana 26:33-52.

Hunt D. 1999. CITES Cactaceae Checklist. Royal Botanical Gardens \& International Organization for Succulent Plant Study, Kew.

IUCN 2003. 2003 Red List of Threatened Species. $<$ http://www.redlist.org $>$

Leirana-Alcocer J. y Parra-Tabla V. 1999. Factors affecting the distribution, abundance and seedling survival of Mammillaria gaumeri, an endemic cactus of coastal Yucatan, México. Journal of Arid Environments 41:421-428.

Lüthy J.M. 2001. The cacti of CITES. Appendix I. CITES, Berna.

Martínez A.J., Suzán A.H. y Salazar O.C. 1994. Aspectos ecológi$\cos$ y demográficos de Neolloydia pseudopectinata (Backeberg) E. F. Anderson. Cactáceas y Suculentas Mexicanas 39:27-33.

Navarro C.M. y Flores-Martínez A. 2002. Aspectos demográficos de Echinocereus pulchellus var. pulchellus en el municipio de Chignahuapan, Puebla. Cactáceas y Suculentas Mexicanas 47:24-32.

Navarro L. y Guitián J. 2003. Seed germination and seedling survival of two threatened endemic species of the northwestern Iberian peninsula. Biological Conservation 109:313-320.

Nobel P.S. 1994. Remarkable Agaves and Cacti. Oxford University Press, Nueva York.

Palmer M.E. 1987. A critical look at rare plant monitoring in the United States. Biological Conservation 39:113-127.

Parker K.C. 1989. Height structure and reproductive characteristics of senita, Lophocereus schotii (Cactaceae) in Southern Arizona. The Southwestern Naturalist 34:392-401.

Pyke D.A. y Thompson J.N. 1986. Statistical analysis of survival and removal rate experiments. Ecology 67:240-245.

Rojas-Aréchiga M. y Vázquez-Yanes C. 2000. Cactus seed germination: a review. Journal of Arid Environments 44:85-104.

Schemske D.W., Husband B.C., Ruckelshaus M.H., Goodwillie C., Parker I.M. y Bishop J.G. 1994. Evaluating approaches to the conservation of rare and endangered plants. Ecology 75:584-606.

SEMARNAP. 2001. Norma Oficial Mexicana. NOM-059-ECOL2001. Diario Oficial de la Federación. 2a Sección. México, D.F.

Sosa V.J. y Fleming T.H. 2002. Why are columnar cacti associated with nurse plants? En: Fleming T.H. y Valiente-Banuet A. Eds. Columnar Cacti and their Mutualists. Evolution, Ecology and Conservation, pp. 306-323, The University of Arizona Press, Tucson.

Valverde T., Trejo M.L. y Castillo S. 1999. Patrón de distribución y abundancia de Mammillaria magnimamma en la reserva del Pedregal de San Ángel, México D.F. Cactáceas y Suculentas Mexicanas 44:64-74.

Fecha de recepción:14 de junio de 2004

Versión corregida: 8 de septiembre de 2004

Aceptado: 14 de septiembre de 2004 